\title{
A Novel Smart Growth Evaluation Model by Applying RBFNN
}

\author{
Jiaxing Zhang \\ North China Electric Power University, Baoding 071000, China. \\ jiaxingzhang_ncepu@163.com
}

Keywords: Smart Growth, AHP Model, Back Propagation Neural Network, RBF Neural Network.

\begin{abstract}
In order to make cities can carry more population, save more resources and maintain rapid development, smart growth based on the three E's of sustainability and the 10 principles comes into being and is considered by many governments. In this paper, we concentrate on measure the success of smart growth and make plans. Firstly, we chose Arlington County and Canberra to research, then select 12 kinds of factors such as green space ratio preliminarily, which can reflect all aspects of the city. After that, we build the Entropy Method and chose the factors whose weight are highest 6 . Next, we formulate the AHP Model to get the remained 6 factor's weight, and can also gain the approximate value of the metric. Then we build the BP Model to develop an accurate formula, but it traps into local extremum many times. Therefore, we use the more optimal model--the RBF Model, whose input is the above 6 factors and output is the value of the metric. To rank the 6 factors, we analyze the sensitivity of our models, and formulate the most suitable plan for cities' development based on the ranks, cities' unique characteristics including geographical conditions, local government expenditure, etc. At last, we proposed a novel method to evaluate a city fits in smart growth or not.
\end{abstract}

\section{Introduction}

The world is rapidly urbanizing. The coming decades will bring further profound changes to the size and spatial distribution of the global population. The continuing urbanization and overall growth of the world's population is projected to add 2.5 billion people to the urban population by 2050 , with nearly 90 percent of the increase concentrated in Asia and Africa. At the same time, the proportion of the world's population living in urban areas is expected to increase, reaching 66 percent by 2050[1]. And sprawling development patterns have become the leading land-use concern in the United States [2]. Figure 1 shows some different forms of sprawl. We can find that different densities will cause different urban patterns, while some patterns are not suitable for the future population distribution and won't be conducive to build resource-conservation societies.

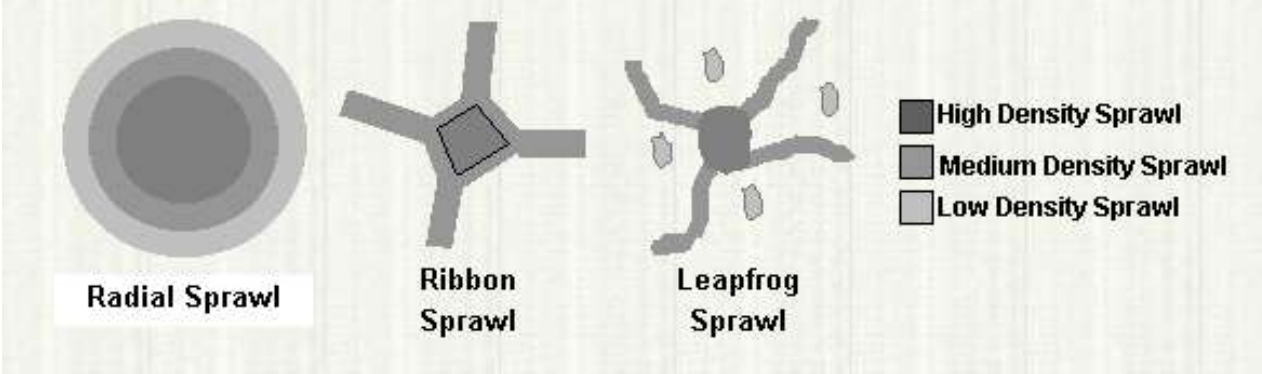

Fig. 1 Forms of Sprawl

In order to make our city can carry more population and save more resources, smart growth come into being. Smart growth is an approach to development that encourages a mix of building types and uses, diverse housing and transportation options, development within existing neighborhoods, and community engagement [3]. It is an urban planning and transportation theory that concentrates growth in compact walkable urban centers to avoid sprawl. It also involves transit-oriented, walkable, bicycle-friendly land use, including neighborhood schools, complete streets, and mixed-use development with a range of housing choices [4]. 
The initial parameters we collected including all aspects about our city. Then we use entropy method to finish our feature selection work, and use AHP to optimize the weight of the final parameters. After selecting parameters that we will use, we first develop a Back Propagation (BP) Neural Network, but it traps into local extremum many times. As a result, we develop a Radial Basis Function (RBF) Neural Network Model to evaluate the success of smart growth of a city. According to our theory and model, we evaluate cities' planning and put forward a more reasonable plan for them. Next, we analyze the sensitivity of main parameters of the model, and rank the individual initiatives within your redesigned smart growth plan as the most potential to the least potential. Afterwards, we explain our growth plan's ways to support a large population growth. At last, we analyze our model's strengths and weaknesses and look forward to the future of modeling which apply to evaluate the smart growth.

\section{Methodology}

\subsection{Entropy Method for Feature Selection}

Firstly, we select a series of variables as our candidate. Then we use Entropy Method [5] to finish our feature selection work. Table 1 shows the initial parameters we selected.

In this part, we select our data form Arlington Count Profile [6]. From this website, we can attend to some basic information of Arlington Count. At the meantime, Arlington is an outstanding representative of the city that implement smart growth. So that it will be an excellent choice for us.

By calculating weight of each parameter, we select the highest weight of different aspect as our basic parameters of our modeling.

At last we select six parameters as our basic parameters for modeling, table 1 shows the parameters we selected.

Table 1. SIX PARAMETERS

\begin{tabular}{c}
\hline Parameters \\
\hline Green Space Ratio \\
high school degree ratio \\
Employment Rate \\
Bus miles/ Population \\
Density of Population \\
GFA/Land Area \\
\hline
\end{tabular}

\subsection{AHP}

By applying Entropy Method, we can get a weight for each parameter that we will use for further modeling. However, we want to get more accurate weight of each parameter, so we use Analytic Hierarchy Process (AHP) [7] to calculate the weight of the parameters that we choose by Model I for optimal results.

In previous work, we have already chosen six parameters as our evaluation indexes. Then we follow some rules and grade in Analytic Hierarchy Process (AHP).

We calculate the relative weight of the rule layer according to the comparative index for judging matrix, and get the largest eigenvalue of comparative matrix. Then we use (1) to calculate coherent deviation index.

$C I=\frac{\lambda_{\max }-n}{n-1}$

Then we combine with the mean random consistency index RI (RI can be achieved by turning to some references) to calculate random conformance rate (C.R.)

$$
C R=\frac{C I}{R I}
$$

If $\mathrm{CR}<0.1$, it means the consistency of the indicators which under the same category of indicators consistency is quite good. Otherwise it behaviors bad, we need to change the categories of our indicators, until we get the consistency we satisfied. 
Through the calculation of the total index of the model index of each level synthesis weights, we synthesis of the weight of each indicator according to the hierarchy of the model. Table 2 shows the weight of each parameter.

Table 2 weight of 6 parameters

\begin{tabular}{cc}
\hline Parameters & Weight \\
\hline Green Space Ratio & 0.0632 \\
high school degree ratio(Perk-12) & 0.3624 \\
Unemployment Rate & 0.0651 \\
Bus miles/ Population & 0.1928 \\
Density of Population & 0.2918 \\
GFA/Land Area & 0.0246 \\
\hline
\end{tabular}

\subsection{Evaluation Model Based on Back Propagation Neural Network}

The BP, one of the most popular techniques in the filed of NN, is a kind of supervised learning neural network, the principle behind which involves using the steepest gradient descent method to reach any small approximation.[8,9]Assume that there are $\mathrm{n}$ input neurons, $\mathrm{m}$ hidden neurons, and one output neuron, we can infer a training process described by the following equations to update these weighted values.

In order to evaluate our BP Neural Network model, we select the city that in line with the smart growth. Then we use the data of a few years ago and its annual evaluation of success of smart growth as the training data of our model. After training work, we use the model which have been trained to predict a few years' evaluation to check the relative error, and the result of 0.1137 shows our model's accuracy is not so good, so next, we decide to use RBF Neural Network Model to improve the evaluation result of our model.

\subsection{Improved Evaluation Model Based on RBF Neural Network}

In our previous work, we finished the feature selection and weight calculation by using Entropy Method and AHP. Next, we decide develop evaluation model by using BP Neural Network to optimize the result of our model. And RBF [10] is a kind of forward network based on the function approximation theory.

After applying RBF neural network, we use the value of the output layer to evaluate the success of smart growth and we completed our evaluation system and modeling work.

In order to evaluate our model, we select the city that in line with the smart growth. Then we use the data of a few years ago and its annual evaluation of success of smart growth as the training data of our model. After training work, we use the model which have been trained to predict a few years' evaluation to check the relative error, and the result of 0.0267 shows our model's accuracy is quite good comparing to our result of BP neural network model's 0.1137

\section{Experiment and Result}

By querying the selected city governments' official website, we access to government report for the current urban planning data, and according to the content of the plan for we calculate the parameters we need in our model. The result of evaluation of two cities' growth is shown in figure 2.

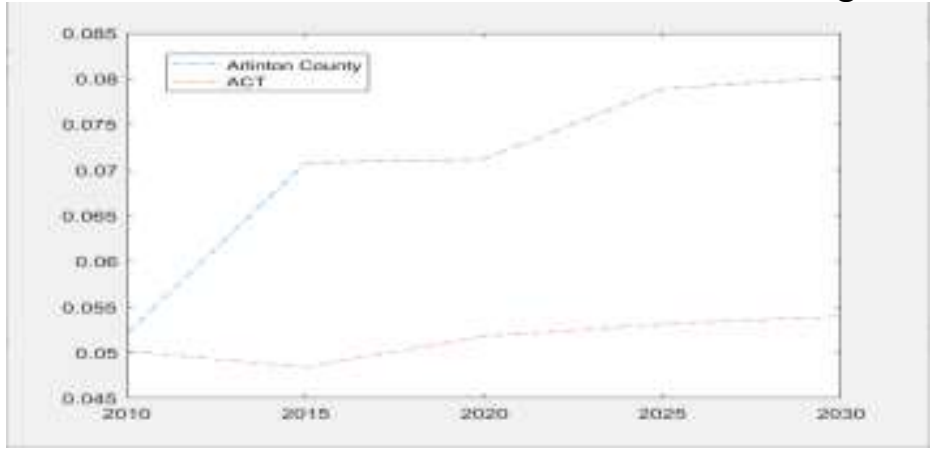

Fig. 2 Smart growth rate of two cities 


\section{Summary}

Smart growth will be a hot topic worth to discuss in social research. In the process of developing a smart city, we need more smart growth on the theoretical research and practical application, so the establishment of a more comprehensive intelligent growth evaluation system is essential. Through regression fitting and other methods, we could predict the next few decades' value of every factor under government current plans and our improving plans. So as to we get the next few decades value of metric with the RBF. Our model and plans have so wide applicability that it could estimate the totally different smart growth city, and solve the situation well when population increase by $50 \%$. Furthermore, it could be of high credibility and high usability, its judging criteria stands for the basic survey of a city and it is objective, simple and comprehensive.

\section{References}

[1]. World Urbanization Prospects, 2014 revision, ISBN 978-92-1-151517-6,

[2]. Freilich, R. (1999). From Sprawl to Smart Growth: Successful Legal, Planning, and Environmental Systems

[3]. Smart Growth America. Information on: https://smartgrowthamerica.org/

[4]. Boeing; et al. (2014). "LEED-ND and Livability Revisited". Berkeley Planning Journal. 27: 31-55. Retrieved 2015-04-15.

[5]. Study on Evaluation of Regional Innovation Capacity in China Based on Entropy Method [J]. Science and Technology Management Research, 2013, 33 (23): 56-61.

[6]. Information on: https://projects.arlingtonva.us/data-research/

[7]. Comprehensive evaluation of the construction level of intelligent city [D]. North China Electric Power University (Beijing), North China Electric Power University, 2012.

[8]. No-neural network method for multi-index comprehensive evaluation [J], 2006 (3): 61-62.,

[9]. Modern Management Science

[10]. Stock market prediction of S\&P 500 via combination of improved BCO approach and BP neural network

[11]. Application of RBF and GRNN Neural Network Model in River Health Assessment - A Case Study of Middle and Small Rivers Health Evaluation in Wenshan Prefecture [J]. China Rural Water and Hydropower, 2012 (3): 56-61. 